\title{
Relationship between spiral and ferromagnetic states in the Hubbard model in the thermodynamic limit
}

\author{
Ryotaro Arita and Hideo Aoki \\ Department of Physics, University of Tokyo, Hongo, Tokyo 113-0033, Japan
}

(November 27, 2018)

\begin{abstract}
We explore how the spiral spin(SP) state, a spin singlet known to accompany fully-polarized ferromagnetic (F) states in the Hubbard model, is related with the $\mathrm{F}$ state in the thermodynamic limit using the density matrix renormalization group and exact diagonalization. We first obtain an indication that when the F state is the ground state the SP state is also eligible as the ground state in that limit. We then follow the general argument by Koma and Tasaki [J. Stat. Phys. 76, 745 (1994)] to find that: (i) The SP state possesses a kind of order parameter. (ii) Although the SP state does not break the $\mathrm{SU}(2)$ symmetry in finite systems, it does so in the thermodynamic limit by making a linear combination with other states that are degenerate in that limit. We also calculate the oneparticle spectral function and dynamical spin and charge susceptibilities for various $1 \mathrm{D}$ finite-size lattices. We find that the excitation spectrum of the SP state and the F state is almost identical. Our present results suggest that the $\mathrm{SP}$ and the $\mathrm{F}$ states are equivalent in the thermodynamic limit. These properties may be exploited to determine the magnetic phase diagram from finite-size studies.

PACS numbers: 71.10.-w,71.10.Fd
\end{abstract}

\section{INTRODUCTION}

The itinerant ferromagnetism has been one of the most fundamental problems in condensed matter physics, and we are still some way from a full understanding of the problem. For the ferromagnetism in the Hubbard model, a simplest possible model for interacting itinerant electrons, there are various studies, which are pioneered by Gutzwillerd, Hubbarde, and Kanamoril . For the Hubbard model on finite-size systems, it recently extended to rigorous proofs that spin-independent Coulomb interaction can indeed result in fully-polarized ferromagnetic ground states without degeneracy for appropriate conditions and/or appropriate models 12 . Apart from these, there is a body of numerical studies for finite systems, where various authors have shown that finite Hubbard models with appropriate band fillings and boundary conditions have fully-polarized ferromagnetic ground states 1216 .

On the other hand, for infinite systems, the itinerant nature of electrons makes the problem interesting and subtle. The most important reason is the existence of the spiral-spin(SP) state, a spin-singlet state that has the min-pin correlation length as large as the sample size 17 19, which is known to accompany the fully-polarized ferromagnetic(F) state in various finitesize itinerant models. By accompanying we mean SP and $\mathrm{F}$ states are close in energy in finite systems, where the ground state changes between them when the boundary condition is twisted.

The SP state does not seem to be an accident of the Hubbard model, since SP states are found not only in electron systems with strong charge-charge interactions, but also in those with spin-dependent interactions such as double-exchang 20 or Kondo lattice 21 models. So we believe that it is a general feature of strongly correlated systems for the $\mathrm{F}$ state to be accompanied by the SP state. It is rather surprising, since the latter has the total $S=0$ while the former has $S=S_{\max }$. Thus it is desirable to resolve this question to understand the ferromagnetism in itinerant systems in the thermodynamic limit. Thus the problem we have to clarify is the relation between the SP and F states in the thermodynamic limit (do those states degenerate or coalesce, etc), which we address in this paper.

First, we show that the SP state is eligible as the ground state (i.e., degenerate with the $\mathrm{F}$ state in energy in the thermodynamic limit) when the $\mathrm{F}$ state is the ground state. We check this for one-dimensional (1D) $t-t^{\prime}$ Hubbard model with infinitely large coulomb interaction taken as a typical example and calculate the energies of the SP and F states as a function of the inverse system size with the density matrix renormalization group (DMRG) method.

Next, we discuss whether the symmetry of the SP state is broken in the thermodynamic limit, following the arguments by Koma and Tasaki22 on symmetry breaking and finite-size effects in quantum many-body systems on a lattice. We show that (i) the SP state possesses a kind of order parameter, (ii) although the state does not break the global $\mathrm{SU}(2)$ symmetry in the finite system, it does so in the thermodynamic limit by making a linear combination of other states that are degenerate in that limit.

We have studied excitation spectrum of the SP state by calculating one-particle spectral function, dynamical spin and charge susceptibilities for the SP state in various finite-size models, including 1D $t-t^{\prime}$ Hubbard model, Tasaki's flat band model, and Hubbard ladder. We have found that the excitation spectrum of these two states in the finite size system are almost identical. 
The present results suggest that we should regard the SP state as identical with the F state although they have the total spins in the opposite extremes $(S=0$ for the SP state, $S=S_{\max }$ for $\mathrm{F}$ ) in the finite size system. These properties can be exploited to determine the magnetic phase diagram from the calculation of the total spin of the finite-size system in an unambiguous fashion. Otherwise we have to worry about not only the total spin, but the spin-spin correlation to identify SP states in determining the phase diagram.

The paper is organized as follows. In Sec. II, we first confirm that the SP and F states have degenerate energy for $L \rightarrow \infty(L$ : sample size $)$ in that the energy per site is identical between them. DMRG is used to calculate the difference in the total energy, $\Delta E \equiv E_{\mathrm{SP}}-E_{\mathrm{F}}$, between the SP state and the $\mathrm{F}$ state as a function of system size $L$ for the $t$ - $t^{\prime}$ Hubbard model with $U=\infty$ as a typical example. The extrapolate $\Delta E(L \rightarrow \infty)$ is always finite (or zero depending on the boundary condition even in the thermodynamic limit), i.e., $\Delta E$ per site does indeed vanish. It implies that when the $\mathrm{F}$ state is the ground state, the SP state is also eligible as the ground state if these states are translation invariant. Then we move on to the discussion on symmetry breaking of the SP state along the Koma-Tasaki's argument. In Sec. III, we calculate the single-particle spectral function and dynamical spin and charge susceptibilities for various models. Our results suggest that the $\mathrm{SP}$ state and the $\mathrm{F}$ state are identical state in the thermodynamic limit.

In Sec. IV, we take an appropriate combination of the boundary condition and the number of electrons to make the SP state higher in energy than the SF state, and the phase diagram for the one-dimensional $t$ - $t^{\prime}$ Hubbard model, Tasaki model, and 2-leg Hubbard ladder model is obtained with exact diagonalization of small systems. We have found that the phase boundaries rapidly converge to those for larger systems obtained with DMRG. We may expect that it can be exploited to the determination of the phase diagram of $2 \mathrm{D}$ or higher dimensional systems. A summary of the present study is given in Sec. VI.

\section{DISCUSSION ON THE THERMODYNAMIC LIMIT}

\section{A. The energy difference between the SP state and the $\mathrm{F}$ state in the thermodynamic limit}

1D $t-t^{\prime}$ Hubbard model is one of the simplest models that is thought to exhibit ferromagnetism for sufficiently strong electron-electron interaction. Müller-Hartmann suggested that in the low-density limit the ground state of $t$ - $t^{\prime}$ Hubard model is ferromagnetid23. Daul and Noack 13 have carried out highly accurate DMRG calculation to conclude that there is an extensive ferromagnetic phase in the phase diagram. Since the $t$ - $t^{\prime}$ Hubbard model is numerically tractable, we study the energy difference of the SP and F states by taking this model with $U=\infty$ as an example.

The $t-t^{\prime}$ Hubbard Hamiltonian is given by

$$
\begin{aligned}
\mathcal{H}= & -t \sum_{i=1}^{L} \sum_{\sigma}\left(c_{i \sigma}^{\dagger} c_{i+1, \sigma}+\text { H.c. }\right) \\
& +t^{\prime} \sum_{i=1}^{L} \sum_{\sigma}\left(c_{i \sigma}^{\dagger} c_{i+2, \sigma}+\text { H.c. }\right)+U \sum_{i=1}^{L} n_{i \uparrow} n_{i \downarrow}
\end{aligned}
$$

where $c_{i \sigma}^{\dagger}$ creates an electron at site $i$ with spin $\sigma(=\uparrow$ $, \downarrow), t$ is the nearest-neighbor hopping, and $t^{\prime}$ the next nearest-neighbor hopping, $U$ the Hubbard repulsion, and $n_{i \sigma} \equiv c_{i \sigma}^{\dagger} c_{i \sigma}$. In this section we take $t=1, t^{\prime}=0.2$ and $U=\infty$.

As we can check by a simple exact diagonalization, the ground state of the system has always $S_{\text {tot }}=0$ for any value of $U$ at least for system sizes up to 12 sites when a periodic boundary condition(PBC) is adopted. This is to be contrasted with the phase diagram obtained by Daul and Noack with an open boundary condition(OBC), in which a wide ferromagnetic region is found for $t^{\prime}=0.2$ and large $U$.

Let us first confirm that the SP and F states are degenerate in the sense that their difference in the total energy behaves as

$$
\Delta E \equiv E_{\text {singlet }}-E_{\mathrm{F}} \rightarrow \text { finite }
$$

i.e., the energy per site vanishes,

$$
\Delta E / L \rightarrow 0
$$

where $E_{\text {singlet }}$ is the lowest energy within the $S_{\text {tot }}=0$ sector, namely the energy of the SP state.

We take OBC, because the DMRG becomes most accurate for this condition. As Daul and Noack showed 13 .14, the ground state of $t-t^{\prime}$ Hubbard model is ferromagnetic for $t^{\prime}=0.2$ and $U \rightarrow \infty$ in OBC at least up to 50 sites. To estimate $\Delta E$, we must calculate the energy of the ground state of the $S_{\text {tot }}=0$ sector which has higher energy than the ferromagnetic ground state. Therefore, we add the term $\lambda \mathbf{S}_{\text {tot }}^{2}$ to the original Hamiltonian (11) to selectively shift the states with higher total spin to higher energies by turning on $\lambda>0$, while conserving the SU(2) symmetry. Here we set $\lambda=1$. In Fig. 1, we show the spin correlation function, $\left\langle S_{i}^{z} S_{j}^{z}\right\rangle(i=L / 2, j=L / 2+1, \cdots, L-3)$ of the spin singlet state for $L=32$ sites, $n=0.75$ as an example. We can see that the spin correlation wave length is as large as the system size. Namely, the spin singlet state is the SP state.

Since DMRG is a variational procedure, the energy of the SP state is an upper bound, while the energy of the $\mathrm{F}$ state is calculated exactly because the $\mathrm{F}$ state does not feel the on-site coulomb interaction. Hence we overestimate $\Delta E$. To minimize this overestimation, we must calculate the energy of the SP state as accurately as possible. This is the reason why we have set $U=\infty$ and 
$t^{\prime}=0.2$. Namely, the exclusion of double occupancies reduces the Hilbert space drastically, while a small value of $\left|t^{\prime}\right|$ reduces the truncation error.

Using the finite-size algorithm in DMRG, we calculated up to $L=48$ for the density of electrons $n=0.5$, sweeping the system about 20 times to improve the wave function. We store the density matrix at each step to construct good initial vector for each super-block diagonalization. We have kept up to $m=500$ states per block at each step, where the convergence is checked by comparing the results for $m=300,400,500$. The truncation error is smaller than $10^{-5}$, which is small enough to enable us to extrapolate $\Delta E(L \rightarrow \infty)$.

In Fig. 2(solid line), we show the results for $\Delta E \equiv$ $E_{\mathrm{SP}}-E_{\mathrm{F}}$ as a function of inverse system size $1 / L$ for electron density $n=0.5$. We can see that all the points for $L>24$ fall upon a linear dependence on $1 / L$, from which we can extract $\Delta E(L \rightarrow \infty)$. For all the densities studied, $\Delta E(L \rightarrow \infty)$ indeed remains finite $\left(\sim 10^{-3}\right)$.

In order to check whether the result is not an accident for $U=\infty$, we can introduce the effect of large but finite $U$ as an (antiferromagnetic) exchange interaction $J>0$,

$$
J \sum_{i=1} P_{G}^{-1}\left(\mathbf{S}_{\mathbf{i}} \cdot \mathbf{S}_{\mathbf{i}+\mathbf{1}}-\frac{1}{4} n_{i} n_{i+1}\right) P_{G},
$$

added to the $U=\infty t-t^{\prime}$ Hubbard model. Here $S_{i} \equiv$ $\frac{1}{2} \sum_{\beta \gamma} c_{i \beta}^{\dagger} \vec{\sigma}_{\beta \gamma} c_{i \gamma}$ is the spin operator, where $\vec{\sigma}$ is the Pauli matrices and $P_{G}$ denotes the Gutzwiller projection operator. In Fig. 2 the results for $J= \pm 0.2$ are superposed. We can see that $\Delta E$ does not change drastically even if we introduce the effect of finite $U$.

This kind of $\Delta E$ may depend on the boundary condition, which can be indeed the case with finite systems. For instance, Kusakabe and Aokil 18 have shown for a finite two-dimensional Hubbard model that Nagaoka's ferromagnetic state (one hole in the half-filled band with $U=\infty$ ) alternate with an SP state as the boundary condition is changed from periodic to anti-periodic. In other words a level crossing takes place between the two states as an Aharonov-Bohm magnetic flux is introduced to twist the boundary condition. Such a boundary effect can affect the 'energy gap' even in the thermodynamic limit in general.

Thus we compare in Fig. 3 the ordinary OBC (the same as Fig. 2, solid line) and the boundary condition in which we further turn off the nearest-neighbor transfers $(t)$ at either end of the system in the $t-t^{\prime}$ model for $\Delta E(L \rightarrow \infty)$ at $n=0.5$. We can see that $\Delta E(L \rightarrow \infty)$ becomes zero (negligibly small) in the latter case. Therefore, we can not exclude a possibility for which the SP state has a lower energy than the F state in some appropriate boundary condition. In other words, the problem which state has a lower energy is a very subtle problem.

\section{B. The definition of the ground state}

As we have seen, it is meaningless in the thermodynamic limit to identify the ground state by studying small 'finite' differences in the total energy between the candidates, so that a totally different point of view is required. The mathematical definition of the ground state in the thermedynamic limit is reviewed in Koma and Tasaki's article 22 and here we follow them. For simplicity, we focus on the case of PBC.

We first recapitulate the definition. Let $A$ be an arbitrary local operator that acts on a finite number of sites. We define $\rho$ as

$$
\rho(A) \equiv \lim _{\Lambda \uparrow Z^{d}} \rho_{\Lambda}(A)
$$

for each $A$, and we call $\rho$ as 'state' in the thermodynamic limit. Here $\Lambda$ is a finite $(L \times L \times \cdots L \times L) d$-dimensional hypercubic lattice while $Z^{d}$ is an infinite $d$-dimensional lattice, and we have defined

$$
\rho_{\Lambda}(\cdots) \equiv \operatorname{Tr}_{\mathrm{H}_{\Lambda}}\left[(\cdots) \tilde{\rho}_{\Lambda}\right]
$$

where the trace is taken over the Hilbert space $\mathrm{H}_{\Lambda}$ spanned over $\Lambda$, and $\tilde{\rho}_{\Lambda}$ is an arbitrary density matrix on $\mathrm{H}_{\Lambda}$. We also assume a Hamiltonian $\mathcal{H}_{\Lambda}$ that is local as defined by

$$
\mathcal{H}_{\Lambda}=\sum_{x \in \Lambda} h_{x}
$$

where $h_{x}$ is a local component such as $h_{x}=$ $\sum_{\sigma}\left(c_{x \sigma}^{\dagger} c_{x+1, \sigma}+\right.$ H.c. $)+U n_{x \uparrow} n_{x \downarrow}$ for the Hubbard model. We then define the ground-state energy density $\varepsilon_{0}$ as

$$
\varepsilon_{0} \equiv \lim _{\Lambda \uparrow Z^{d} \Phi_{\Lambda} \in \operatorname{Hnf}_{\Lambda},\|\Phi\|=1} \frac{1}{|\Lambda|}\left\langle\Phi_{\Lambda}\left|\mathcal{H}_{\Lambda}\right| \Phi_{\Lambda}\right\rangle,
$$

where $\Phi$ is a state in $\mathcal{H}_{\Lambda}$ and $|\Lambda|$ is the norm of $\Lambda$. A state $\omega$ in the infinite-volume limit is said to be a ground state if it satisfies

$$
\omega\left(h_{x}\right)=\varepsilon_{0}
$$

for arbitrary $x$.

In the present context, we have seen in the previous section that the numerical results for the Hubbard model imply that the energy per site of the SP state and the $\mathrm{F}$ state is identical in the thermodynamic limit for any boundary condition, especially PBC. In this boundary condition the system has a translational invariance, so that an SP state in a finite system should have

$$
T(x)|\mathrm{SP}\rangle_{\Lambda}=\exp (i \gamma)|\mathrm{SP}\rangle_{\Lambda}
$$

under the translation $T(x)$ which translates the state by $x$. We can expect that the invariance holds in the thermodynamic limit as well, so that 


$$
\begin{aligned}
\left\langle\mathrm{SP}\left|h_{x_{0}+x}\right| \mathrm{SP}\right\rangle & =\left\langle\mathrm{SP}\left|T(x) h_{x_{0}} T^{-1}(x)\right| \mathrm{SP}\right\rangle \\
& =\left\langle\mathrm{SP}\left|h_{x_{0}}\right| \mathrm{SP}\right\rangle \\
& =\varepsilon_{0},
\end{aligned}
$$

and we can conclude that the SP state in PBC is also the ground state in the thermodynamic limit.

\section{Symmetry breaking in the spiral spin state}

One important question is: does the SP state, despite its being spin singlet, accompany a symmetry breaking in the thermodynamic limit? For simplicity, we consider the 1D case. From the numerical results for the spinspin correlation in finite systems, a natural quantity that is expected to become nonzero (in PBC) is

$$
\frac{1}{L^{2}} \sum_{i, j \in \Lambda} \cos \left(\theta_{i}-\theta_{j}\right)\left\langle\mathbf{S}_{i} \cdot \mathbf{S}_{j}\right\rangle_{\mathrm{SP}}>0
$$

where $\theta_{j}=2 \pi j / L$. We can then introduce an order parameter,

$$
\begin{aligned}
O_{\Lambda}^{ \pm} & \equiv \frac{1}{L} \sum_{i \in \Lambda} \exp \left(\mp i \theta_{i}\right) S_{i}^{ \pm} \equiv \frac{1}{L} \sum_{i \in \Lambda} o_{i}^{ \pm}, \\
O_{\Lambda}^{(1)} & =\frac{1}{2}\left(O_{\Lambda}^{+}+O_{\Lambda}^{-}\right) \\
& \equiv \frac{1}{L} \sum_{i \in \Lambda} \cos \theta_{i} S_{i}^{x}+\sin \theta_{i} S_{i}^{y}, \\
O_{\Lambda}^{(2)} & =\frac{1}{2 i}\left(O_{\Lambda}^{+}-O_{\Lambda}^{-}\right) \\
& \equiv \frac{1}{L} \sum_{i \in \Lambda}-\sin \theta_{i} S_{i}^{x}+\cos \theta_{i} S_{i}^{y}, \\
O_{\Lambda}^{(z)} & \equiv \frac{1}{L} \sum_{i \in \Lambda} S_{i}^{z}
\end{aligned}
$$

where $S^{ \pm}=S^{x} \pm i S^{y}$. Three operators $O_{\Lambda}^{(1)}, O_{\Lambda}^{(2)}, O_{\Lambda}^{(z)}$, which obey a Lie algebra, satisfy the assumption required for Theorem 2.4 in Ref. 22. The expectation values of the squared $O_{\Lambda}^{(1)}$ is

$$
\begin{aligned}
{ }_{\Lambda}\left\langle\mathrm{SP}\left|O_{\Lambda}^{(1) 2}\right| \mathrm{SP}\right\rangle_{\Lambda}= & \frac{2}{3 L^{2}} \sum_{i, j \in \Lambda} \cos \left(\theta_{i}-\theta_{j}\right)\left\langle\mathbf{S}_{i} \cdot \mathbf{S}_{j}\right\rangle_{\mathrm{SP}} \\
& +\frac{1}{2 i L^{2}} \sum_{i, j \in \Lambda} \sin \left(\theta_{i}-\theta_{j}\right)\left\langle S_{i}^{+} S_{j}^{-}\right\rangle_{\mathrm{SP}} .
\end{aligned}
$$

We can always take $|\mathrm{SP}\rangle_{\Lambda}$ to be real in $\mathrm{PBC}$ since $\mathcal{H}_{\Lambda}$ is real. $S_{i}^{ \pm}$can also be represented as a real matrix, so that $\left\langle S_{i}^{+} S_{j}^{-}\right\rangle_{\mathrm{SP}}$ is a real number, and we have $\left\langle S_{i}^{+} S_{j}^{-}\right\rangle_{\mathrm{SP}}=\left\langle S_{j}^{+} S_{i}^{-}\right\rangle_{\mathrm{SP}}$ so that the last line above vanishes. From the inequality eq.(2) we have

$$
\begin{aligned}
{ }_{\Lambda}\left\langle\mathrm{SP}\left|O_{\Lambda}^{(1) 2}\right| \mathrm{SP}\right\rangle_{\Lambda} & ={ }_{\Lambda}\left\langle\mathrm{SP}\left|O_{\Lambda}^{(2) 2}\right| \mathrm{SP}\right\rangle_{\Lambda} \\
& =\frac{2}{3 L^{2}} \sum_{i, j \in \Lambda} \cos \left(\theta_{i}-\theta_{j}\right)\left\langle\mathbf{S}_{i} \cdot \mathbf{S}_{j}\right\rangle_{\mathrm{SP}}>0 .
\end{aligned}
$$

On the other hand the order parameters, when not squared, satisfy

$$
{ }_{\Lambda}\left\langle\mathrm{SP}\left|O_{\Lambda}^{(1)}\right| \mathrm{SP}\right\rangle_{\Lambda}={ }_{\Lambda}\left\langle\mathrm{SP}\left|O_{\Lambda}^{(2)}\right| \mathrm{SP}\right\rangle_{\Lambda}=0 .
$$

Thus we have

$$
\Psi_{\Lambda}^{M} \equiv \frac{\left(O_{\Lambda}^{+}\right)^{M}|\mathrm{SP}\rangle_{\Lambda}}{\|\left(O_{\Lambda}^{+}\right)^{M}|\mathrm{SP}\rangle_{\Lambda} \|}
$$

as the 'low-lying state' in the Theorem 2.4 in Ref. 22, which asserts that a symmetry breaking can occur when some low-lying excitations whose energies approach that of the state in question like $1 / L$ mix with it. Here $\Psi_{\Lambda}^{M}$ does have an energy that approaches to $E_{\mathrm{SP}}$ as

$$
\left|\left(\Psi_{\Lambda}^{M}, H_{\Lambda} \Psi_{\Lambda}^{M}\right)-E_{\mathrm{SP}}\right| \leq \text { const. } \times\left(\frac{M^{2}}{L}\right) .
$$

We also have, according to Theorem 2.522,

$$
\lim _{k \rightarrow \infty} \lim _{\Lambda \uparrow Z}\left(\Xi_{\Lambda}^{k}, O_{\Lambda}^{(1)} \Xi_{\Lambda}^{k}\right)=\text { const. }>0
$$

where,

$$
\Xi_{\Lambda}^{k} \equiv \frac{1}{\sqrt{2 k+1}}\left[|\mathrm{SP}\rangle_{\Lambda}+\sum_{M=1}^{k}\left(\Psi_{\Lambda}^{M}+\Psi_{\Lambda}^{-M}\right)\right] .
$$

Here, let us introduce the translation operator $T(x)$, which satisfies $T(x) c_{x_{0}}^{\dagger} T^{-1}(x)=c_{x_{0}+x}^{\dagger}$. Since

$$
\begin{aligned}
T(x) O_{\Lambda}^{+} T^{-1}(x) & =\frac{1}{L} \sum_{x_{0} \in \Lambda} \exp \left(-i \theta_{x_{0}}\right) T(x) S_{x_{0}}^{+} T^{-1}(x) \\
& =\frac{1}{L} \sum_{x_{0} \in \Lambda} \exp \left(-i \theta_{x_{0}}\right) S_{x+x_{0}}^{+} \\
& =\exp \left(i \theta_{x}\right) O_{\Lambda}^{+},
\end{aligned}
$$

$T(x) \Psi_{\Lambda}^{M}=\exp \left(i M \theta_{x}\right) \exp (i \gamma) \Psi_{\Lambda}^{M}$, where $\gamma$ is a constant. Thus, for $o_{i}^{+} \equiv \mathrm{e}^{-i \theta_{i}} S_{i}^{+}$appearing in eq. (3),

$$
\begin{aligned}
{ }_{\Lambda}\left\langle\Psi_{\Lambda}^{M}\left|o_{x+x_{0}}^{+}\right| \Psi_{\Lambda}^{M-1}\right\rangle_{\Lambda}= & { }_{\Lambda}\left\langle\Psi_{\Lambda}^{M}\left|\mathrm{e}^{-i \theta_{x+x_{0}}} S_{x+x_{0}}^{+}\right| \Psi_{\Lambda}^{M-1}\right\rangle_{\Lambda} \\
= & { }_{\Lambda}\left\langle\Psi_{\Lambda}^{M}\left|\mathrm{e}^{-i \theta_{x}} T(x) o_{x_{0}}^{+} T^{-1}(x)\right| \Psi_{\Lambda}^{M-1}\right\rangle_{\Lambda} \\
= & \mathrm{e}^{\left(-i \theta_{x}+i M \theta_{x}-i(M-1) \theta_{x}+i \gamma-i \gamma\right)} \\
& \times{ }_{\Lambda}\left\langle\Psi_{\Lambda}^{M}\left|o_{x_{0}}^{+}\right| \Psi_{\Lambda}^{M-1}\right\rangle_{\Lambda} \\
= & { }_{\Lambda}\left\langle\Psi_{\Lambda}^{M}\left|o_{x_{0}}^{+}\right| \Psi_{\Lambda}^{M-1}\right\rangle_{\Lambda} .
\end{aligned}
$$

Therefore, we have

$$
\begin{aligned}
\lim _{k \rightarrow \infty} \lim _{\Lambda \uparrow Z} \frac{1}{|\Omega|} \sum_{x \in \Omega}\left\langle\Xi_{\Lambda}^{k}\left|o_{x}^{+}\right| \Xi_{\Lambda}^{k}\right\rangle & =\lim _{k \rightarrow \infty} \lim _{\Lambda \uparrow Z} \frac{1}{|\Omega|} \sum_{x \in \Omega}\left\langle\Xi_{\Lambda}^{k}\left|o_{x+x_{0}}^{+}\right| \Xi_{\Lambda}^{k}\right\rangle \\
& =\text { const. }>0,
\end{aligned}
$$

where $\Omega$ is a finite subspace of $\Lambda$ whose size is $|\Omega|$. Hence, 


$$
\lim _{k \rightarrow \infty} \lim _{\Lambda \uparrow Z} \frac{1}{|\Omega|} \sum_{x \in \Omega}\left\langle\Xi_{\Lambda}^{k}\left|S_{x}^{+}\right| \Xi_{\Lambda}^{k}\right\rangle=\text { const. }>0
$$

Namely, although the SP state itself does not break the symmetry, it does so in in the thermodynamic limit by letting $\left\{\Psi_{\Lambda}^{M}\right\}$ mix with it in that limit. In other words, an arbitrarily large but finite region in an SP state has a broken $\mathrm{SU}(2)$ with a finite magnetization.

If we use identities $S^{-}=\sum_{x_{i} \in \Lambda} S_{i}^{-},\left[O^{-}, S^{-}\right]=0$, $\langle\mathrm{SP}| S^{-}=0$,

$$
\begin{aligned}
& \left\langle\Psi_{\Lambda}^{M} \mid \mathrm{F} ; S^{z}=M\right\rangle \\
& ={ }_{\Lambda}\left\langle\mathrm{SP}\left|\left(O_{\Lambda}^{-}\right)^{M}\left(S^{-}\right)^{S_{\max }-M}\right| \mathrm{F} ; S^{z}=S_{\max }\right\rangle_{\Lambda} \\
& =0
\end{aligned}
$$

Thus both of the SP state and $\left\{\Psi_{\Lambda}^{M}\right\}$ that mixes with it in the thermodynamic limit turn out to be orthogonal to the $\mathrm{F}$ state for finite systems.

Finally, let us discuss whether $\Xi$ is the ground state in the thermodynamic limit by checking whether $\left\langle\Xi\left|h_{x}\right| \Xi\right\rangle=\varepsilon_{0}$ holds for arbitrary $x$. Since

$$
\begin{aligned}
& { }_{\Lambda}\left\langle\mathrm{SP}\left|\left(O_{\Lambda}^{-}\right)^{M} h_{x_{0}+x}\left(O_{\Lambda}^{+}\right)^{M}\right| \mathrm{SP}\right\rangle_{\Lambda} \\
& ={ }_{\Lambda}\left\langle\mathrm{SP}\left|\left(O_{\Lambda}^{-}\right)^{M} T(x) h_{x_{0}} T^{-1}(x)\left(O_{\Lambda}^{+}\right)^{M}\right| \mathrm{SP}\right\rangle_{\Lambda} \\
& ={ }_{\Lambda}\left\langle\mathrm{SP}\left|\left(O_{\Lambda}^{-}\right)^{M} h_{x_{0}}\left(O_{\Lambda}^{+}\right)^{M}\right| \mathrm{SP}\right\rangle_{\Lambda} \\
& =\varepsilon_{0},
\end{aligned}
$$

$\left(\Psi_{\Lambda}^{M}, h_{x} \Psi_{\Lambda}^{M}\right)=\varepsilon_{0}$ does indeed hold for arbitrary $x$ in the limit of $L \rightarrow \infty$. This means $\Xi$ is the ground state.

\section{THE EXCITATION SPECTRUM OF THE SPIRAL SPIN STATE}

Having shed light in the context of Koma-Tasaki's argument, we may now conjecture that the SP state and the F state are identical in the thermodynamic limit. Thus let us have a closer look at the SP state as compared with the $\mathrm{F}$ state in three one-dimensional models, i.e., the $t-t^{\prime}$ Hubbard model, Tasaki's flat band model, and the twoleg Hubbard ladder. To determine whether the SP and $\mathrm{F}$ states are identical in the thermodynamic limit, we must show that the relation $\omega_{\mathrm{SP}}(A)=\omega_{\mathrm{SF}}(A)$ holds for arbitrary local operator $A$. Although it is impossible to confirm this for all the possible $A$, if the $\mathrm{F}$ state and the SP state are identical in that limit, the excitation spectrum of these state in finite size system are expected to be almost identical. To confirm this, we look specifically at the single-particle spectral function and the dynamical spin and charge correlation functions of the SP state. Indeed we will show that since the nature of the SP state in finite systems is almost identical to that of the $\mathrm{F}$ state, we cannot distinguish the SP state from the F state by looking at these quantities for sufficiently large system.

\section{A. $t-t^{\prime}$ Hubbard model}

In the $t$ - $t^{\prime}$ Hubbard model where the electron transfer extends to next nearest neighbors $\left(t^{\prime}\right)$, the ground state is $S_{\text {tot }}=0$ for any value of $U$ in PBC at least for sizes up to 12 sites as mentioned in the previous section. Let us first look at the spin-spin correlation function, $\left\langle\Phi_{G}\left|S_{i}^{z} S_{j}^{z}\right| \Phi_{G}\right\rangle$, to confirm that the $S_{\text {tot }}=0$ ground state for large enough $U \geq U_{C}$ is the SP state. We show in Fig. 4t the result for 10 electrons in a 12-site ring (two holes) with $U=20$ or 40 . For $U=20$, a short-range antiferromagnetic spin-spin correlation is observed for $\left\langle S_{i} S_{i+1}\right\rangle<0$. By contrast, for $U=40$ (or larger, which falls upon the ferromagnetic region in the phase diagram; see Fig. 14 below), the spin-spin correlation has a wave length as large as the system size, which is the same behavior of the lowest $S_{\text {tot }}=0$ state in OBC (Fig. 11).

A more detailed nature the states is encapsulated in the single-particle spectral function given by

$$
A^{ \pm}(k, \omega)=\frac{1}{\pi} \operatorname{Im}\left\langle\Phi_{G}\left|\gamma_{k \sigma}^{\mp} \frac{1}{\omega \pm\left(E_{0}-\mathcal{H}\right)-i 0} \gamma_{k \sigma}^{ \pm}\right| \Phi_{G}\right\rangle,
$$

where $A^{+}\left(A^{-}\right)$denote the electron addition (removal) spectrum with $\gamma_{k \sigma}^{+} \equiv c_{k \sigma}^{\dagger}, \gamma_{k \sigma}^{-} \equiv\left(\gamma_{k \sigma}^{+}\right)^{\dagger},\left|\Phi_{G}\right\rangle$ and $E_{0}$ the ground state and its energy, respectively. We have numerically calculated this quantity. In Fig. 5, we show the result for the parameter values adopted in Fig. 4 with $U=40$. We can see that the Luttinger relation, $k_{F}=\pi n / 2$ (with $n=10 / 12=0.83$ here), for the spin unpolarized electrons is violated in favor of $k_{F}=\pi n$, which would be expected for fully spin-polarized fermions. This behavior in the single particle spectral function is consistent with the nearly ferromagnetic nature of the SP state. Such a behavigr was first found for the $t-t^{\prime}-J$ model by Eder and $9 h t a 24$, and then given an interpretation subsequently25.

The $k$-dependence of the spectral function may be analyzed evoking the argument by Doucot and Wen(DW) 26 , who studied the infinite- $U$ Hubbard model on a two-dimensional square lattice with two holes to find a trial state that gives an energy lower by $1 / L^{2}$ than $\mathrm{Na}$ gaoka's ferromagnetic state. The key idea of DW is based on the following intuition. Holes behave as free fermions for on-site interactions when the background spin state is ferromagnetic (or nearly so). Assume that fully polarized electrons take an open-shell configuration (in which there is a degeneracy in the free-electron configurations) in $k$ space in $\mathrm{PBC}$. If the background spin state is changed from $\mathrm{F}$ to the $\mathrm{SP}$ spin texture, a fictitious gauge field corresponding to half flux quantum is generated. This shifts the $k$-points by half the $k$-point spacing, which lowers the kinetic energy because the polarized electrons now take a closed-shell configuration.

It is a nontrivial question whether the DW's trial state may be applied to reproduce the spinless-fermion-like behavior in $A(k, \omega)$ found here for the 1D system. Let us assume, for simplicity, that the holes hop in a rigid spin 
background, although in the original paper DW considered the holes dressed by spin waves. The energy of the hole in the $t-t^{\prime}$ Hubbard model is then given as

$$
\varepsilon=-2 t \cos \left(k \pm \frac{\pi}{L}\right)+2 t^{\prime} \cos 2\left(k \pm \frac{\pi}{L}\right),
$$

where $k(=2 \pi N / L, 0 \leq N \leq L, N$ is an integer $)$ is wave number and \pm corresponds to the sign of the fictitious flux. We can see that the fitting in Fig. 5 is remarkably good. To be precise, the transfer integrals in the original DW are taken to be effective ones $\left(t \rightarrow t \cos (\pi / L), t^{\prime} \rightarrow t^{\prime} \cos (2 \pi / L)\right)$ to take care of the reduction in the transfer between slightly twisted spins, but the present result is better fitted with these reduction factors omitted (dashed curves).

In the addition spectrum an almost dispersionless band of low intensity peaks is seen (at around $E=2$ in Fig. 5). If the ground state were the fully polarized $\mathrm{F}$ state, then such a band is expected, since we can add an opposite spin at any $k$-point. A remnant of such a band again suggests the nearly ferromagnetic nature of the SP state.

A next question is whether the SP state persists for more than two holes. In Fig. 6, we show the spin-spin correlation for 6 electrons on a 12-site lattice (six holes, or $n=0.5$; quarter filled) in $\mathrm{PBC}$ for $U=4,6$. We can see that for $U=6$, the spin-spin correlation is again spiral. To investigate whether DW's picture is valid for such a high hole doping, we have calculated the single-particle spectrum for $U=6$ in Fig. 7. The spectrum for the SP state is fitted well by the energy dispersion defined by the eq.(10), again $t$ and $t^{\prime}$ not reduced, even though the hole concentration is as large as $n_{h}=0.5$. This is surprising, since the assumption that holes are nearly free would be valid only for small enough doping.

We next question whether the SP state is connected adiabatically to the antiferromagnetic state as we decrease the Hubbard $U$. We show the ground-state energy as a function of $1 / U$ for 6 electrons on a 12 -site ring in Fig. 8. We can clearly identify a level crossing appearing as a cusp around $U \simeq 5$, which indicates that a transition (rather than an adiabatic connection) occurs within the $S=0$ space from the antiferromagnetic phase to the SP phase.

We now turn to the dynamical spin and charge correlation functions

$$
\begin{aligned}
& N(k, \omega)=\frac{1}{\pi} \operatorname{Im}\left\langle\Phi_{G}\left|N_{k} \frac{1}{\omega+\left(E_{0}-\mathcal{H}\right)-i 0} N_{k}\right| \Phi_{G}\right\rangle, \\
& S(k, \omega)=\sum_{\alpha} \frac{1}{\pi} \operatorname{Im}\left\langle\Phi_{G}\left|S_{k}^{\alpha} \frac{1}{\omega+\left(E_{0}-\mathcal{H}\right)-i 0} S_{k}^{\alpha}\right| \Phi_{G}\right\rangle,
\end{aligned}
$$

where $N_{k}$ and $S_{k}^{\alpha}$ are Fourier transform of $\left(n_{i}-n\right)$ and $S_{i}^{\alpha}(\alpha=x, y, z)$, respectively. In Fig. 9 , we show the results for the F state in APBC and the SP state in PBC for 10 electrons in 12 sites with $U=40$. We can see that the behavior of these functions for the SP state is surprisingly similar to those of the $\mathrm{F}$ state even for $L=12$.
We can expect that they will become identical in the thermodynamic limit.

\section{B. One-dimensional Tasaki model}

Tasaki 19 proposed a Hubbard model on a special class of lattice structures for which the lowest energy band is dispersionless (flat). He proved rigorously that the ground state is ferromagnetic for arbitrary interaction strength $(0<U<\infty)$ when the flat band is half-filled.

To clarify whether metallic ferromagnetism can be realized in models having a non-half-filled, nearly-flat band, Sakamoto and Kubo 16 investigated with DMRG the Hubbard model on a chain of triangles, which may be thought of as a realization of Tasaki's model in 1D. They obtained results which suggest that the system exhibits metallic, fully-polarized ferromagnetism for sufficiently strong interaction. On the other hand, Watanabe and Miyashita 19 found that an SP state is the ground state at least for system sizes up to $L=12$ for appropriate conditions, i.e., sufficiently large $U, n>0.5$, even number of electrons and PBC.

The Hamiltonian is given by

$$
\begin{aligned}
\mathcal{H}= & t \sum_{i=1}^{L / 2} \sum_{\sigma}\left(c_{2 i, \sigma}^{\dagger} c_{2(i+1), \sigma}+\text { H.c. }\right) \\
& +\alpha t \sum_{i=1}^{L / 2} \sum_{\sigma}\left[\left(c_{2 i-1, \sigma}^{\dagger}+c_{2 i+1, \sigma}^{\dagger}\right) c_{2 i, \sigma}+\text { H.c. }\right] \\
& +\beta t \sum_{i=1}^{L / 2} \sum_{\sigma} n_{2 i-1, \sigma}+U \sum_{i=1}^{L} n_{i \uparrow} n_{i \downarrow}
\end{aligned}
$$

where $t$ is the transfer for the bottom bond in a triangle, $\alpha t$ for the bonds connecting bottom and top sites and $\beta t$ the site energy of a top site. Since a unit cell contains two sites, the single-electron spectrum consists of two bands with the dispersion relation, up to a constant,

$$
\varepsilon^{ \pm}=\frac{t}{2}\left[2 \cos k \pm \sqrt{4 \cos ^{2} k+\left(8 \alpha^{2}-4 \beta\right) \cos k+\beta^{2}+8 \alpha^{2}}\right] .
$$

The lower band is then flat when $\beta=\alpha^{2}-2$ is satisfied. Hereafter in this subsection, we take $t=1, \alpha=2, \beta=0$ (for which the lower band is dispersive) and $L$ even.

While Watanabe and Miyashita have shown that SP states appear for more than quarter-filled bands $(0.5<$ $n<1.0$ ), we find here that the SP state is the ground state of $S_{\text {tot }}=0$ sector for sufficiently large $U$ in the region $n \leq 0.5$ as well at least for $L \leq 12$. In Fig. 10, we show the spin correlation for 6 electrons on a 12-site lattice in PBC for $U=4$ and $U=6$. We can see that for the case of $U=6$ the spin correlation length is indeed as large as the system size. We have also calculated the single-particle spectral function, eq.(9), where we take $\gamma_{i, \sigma}^{+}=c_{2 i, \sigma}^{\dagger}$ or $\gamma_{i, \sigma}^{+}=c_{2 i-1, \sigma}^{\dagger}$. If we combine 
them, they should contain the contributions from the two bands. Here $\gamma_{i \sigma}^{+}$is a Fourier transform of $\gamma_{k \sigma}^{+}$. In Fig. 11, we show the result for 6 electrons on 12 site lattice, where the spectrum is fitted by a two-band extension of eq. (10),

$\varepsilon^{ \pm}=t \cos \left(k \pm \frac{\pi}{L}\right) \pm t \sqrt{\cos ^{2}\left(k \pm \frac{\pi}{L}\right)+8 \cos \left(k \pm \frac{\pi}{L}\right)+8}$

Fig. 11 shows that the DW picture (almost free electrons hopping in a twisted spin background) is surprisingly good even for the two-band case.

\section{Two-leg Hubbard ladder}

Ferromagnetic ground state in 1D has also been obtained for ladder systems, where two chains are connected with an inter-chain transfer, $t_{\perp}$. Liang and Pang 15 suggested that for $t_{\perp} / t=1$ and $U=\infty$, the $\mathrm{F}$ state is one of the ground states for $n_{h}<0.22$, while the ground state is spin singlet for $n_{h}>0.4$. Kohnd 12 presented rigorous results for $U=\infty$ 2-leg Hubbard ladder in the limit of large inter-chain hopping $\left(t_{\perp} / t \rightarrow \infty\right)$. He also studied the case for finite values of $t_{\perp} / t$ with DMRG to obtain the phase diagram. His results are consistent with that of Liang and Pang. These studies assumed OBC.

On the other hand, it is intriguing to study whether an SP state appears in the ladders as well for PBC, and if so, whether $A(k, \omega)$ exhibits a DW-like behavior. The Hamiltonian is given by

$$
\begin{aligned}
\mathcal{H}= & -t \sum_{i=1}^{L} \sum_{\alpha, \sigma}\left(c_{i, \alpha \sigma}^{\dagger} c_{i+1, \alpha \sigma}+\text { H.c. }\right) \\
& -t_{\perp} \sum_{i=1}^{L} \sum_{\sigma}\left(c_{i, 1 \sigma}^{\dagger} c_{i, 2 \sigma}+\text { H.c. }\right)+U \sum_{n=1}^{L} \sum_{\alpha} n_{i \alpha \uparrow} n_{i \alpha \downarrow},
\end{aligned}
$$

where $\alpha(=1,2)$ labels the two legs of the ladder. We set $t=1, U=\infty$.

We have calculated the intra-chain spin-spin correlation $\left\langle S_{i \alpha}^{z} S_{j \alpha}^{z}\right\rangle$ (Fig.12), and found that the ground state is indeed SP. We have also calculated the spectral function, eq. (9), where we now take $\gamma_{k \sigma}^{+}=c_{k 1 \sigma}^{\dagger}+c_{k 2 \sigma}^{\dagger}$ (creating an electron in the bonding band) or $\gamma_{k \sigma}^{+}=c_{k 1 \sigma}^{\dagger}-c_{k 2 \sigma}^{\dagger}$ (antibonding). In Fig.13, we show the result for two holes (14 electrons on a $8 \times 2$ ladder). We fit the spectrum by the dispersion,

$$
\varepsilon=-2 t \cos \left(k \pm \frac{\pi}{L}\right) \pm t
$$

Again the picture of two holes hopping in a twisted spin background gives an accurate description.

\section{MAGNETIC PHASE DIAGRAM FROM FINITE-SIZE STUDIES}

Our results in the previous sections suggest that we should regard an SP state as being ferromagnetic rather than non-magnetic even though $S_{\text {tot }}=0$. So we fi(11) hally come to the problem of how to determine the magnetic phase diagram from finite-size studies in the light of the SP state. As we have stressed, the ground state of finite systems in a certain boundary condition always has $S_{\text {tot }}=0$ no matter how $U$ is strong in some onedimensional models as exemplified by the $t-t^{\prime}$ Hubbard model and the Hubbard ladder. Even when a ferromagnetic state appears for finite systems, the magnetic region in the phase diagram can shrink for some system size as shown by Sakamoto and Kubo16 for the 1D Tasaki's model. This is due to the appearance of the SP state, which are encountered if we assume PBC for even number of electrons, or more generally, if we assume a boundary condition for which the fully-polarized electrons take an open-shell configuration in the ground state.

Since the SP state may be regarded as ferromagnetic as elaborated in previous sections, we have then to distinguish the SP state from nonmagnetic states by calculating the spin correlation, etc. Here we propose that this difficulty can be readily overcome by taking an appropriate combination of the boundary condition and the electron number for which the SP state is excluded from the ground state - this enables us to obtain a reliable magnetic phase diagram within finite-size studies by simply looking at $S_{\text {tot }}$ without worrying about the existence of $S_{\text {tot }}=0$ ferromagnetic-like states. We stress here that this procedure is allowed because the SP state may be regarded as ferromagnetic: if the SP state were a distinct state, then the exclusion of SP states would lead to a missed phase transition.

Here we illustrate this with an appropriate boundary condition that makes the F state take a closed-shell configuration. We present the phase diagram thus obtained for the models employed in section III, i.e., 1D $t$ - $t^{\prime} \mathrm{Hub}-$ bard model, Tasaki's model, and 2-leg Hubbard ladder. In all cases, the results accurately coincide with those obtained by DMRG calculation for much larger systems with OBC. In other words, the result converges to the thermodynamic limit rapidly when we concentrate on the F states. We may expect that this method should be applicable to the determination of the phase diagram of $2 \mathrm{D}$ or higher dimensional systems.

\section{A. $t-t^{\prime}$ Hubbard model}

We first observe that, in the one-electron energy band of the $t-t^{\prime}$ Hubbard model,

$$
\varepsilon(k)=-2 t \cos k+2 t^{\prime} \cos (2 k),
$$


the band minimum at $k=0$ splits into double minima for $t^{\prime}>0.25$ as $t^{\prime}$ is increased. Hereafter in this section, we set $t=1$.

We first look at the case of $t^{\prime}=0.2<0.25$. The band has a single minimum, so that an even number of fullypolarized electrons take a closed-shell configuration for all densities $n<1$ if we assume APBC to put the $k$ points symmetrically about $k=0$. In Fig. 14, we show the exact diagonalization results for 4,6 , 8, or 10 electrons in a 12 -site system $(0.33 \leq n \leq 0.83)$, and 4,6 , or 8 electrons in a 10 -site system. We have also plotted the DMRG result for the phase boundary obtained by Daul and Noack for a 50-site system with OBd13,14. We can immediately see that the results obtained with APBC for fairly small systems is close to that obtained for larger ones.

If we move on to the case of a larger $t^{\prime}=0.8$, an even number of fully-polarized electrons only take a closedshell configuration for APBC when the Fermi energy is higher than $-2 t+2 t^{\prime}$. When the Fermi energy is lower, the situation depends on how the $k$-points are located around the double-minimum dispersion in the given boundary condition, so we concentrate on the former case. In Fig. 15, we show the results for 8 or 10 electrons in a 12-site system and 8 electrons in a 10-site system. we can see that the result for small systems with $\mathrm{APBC}$ is very close to the DMRG result for a 50 -site system.

\section{B. One-dimensional Tasaki's model}

The phase diagram against $U$ and $\beta$ (that controls the dispersion of the band) of the 1D Tasaki's model a chain of triangles) was obtained by Sakamoto and Kubo 16 for various band fillings $(n=1 / 2,1 / 4,3 / 8)$ by means of the DMRG method with PBC. As they mention, the phase boundary between $S_{\text {tot }}=S_{\text {max }}$ and $S_{\text {tot }}=0$ varies strongly with the system size for small systems, so that a reliable phase diagram can be obtained only by going to sufficiently large $(L=32)$ systems.

In Fig. 16, we show the phase boundary determined by exact diagonalization for $n=1 / 2$ and system size as small as 8 or 12 sites with APBC. Here we fix $\alpha=2$, so that the lower band becomes flat for $\beta=2$. Again, the result excellently agrees with that of 32 sites with $\mathrm{PBC}$.

\section{Two-leg Hubbard ladder model}

As mentioned in Sec. IIIC, Kohnd 12 obtained the phase diagram of $U=\infty$ Hubbard ladder by means of the DMRG method. According to his results, there is a wide region of partially ferromagnetic phase in the neighborhood of paramagnetic phase.

Let us consider whether we can reproduce the phase boundary between non-magnetic phase and partially po- larized phase by assuming APBC for small systems. We consider the case of 6 and 8 electrons on a $6 \times 2$ lattice and 4 and 6 electrons on a $4 \times 2$ lattice. We performed calculation for $0.125 \leq t_{\perp} /\left(t_{\perp}+t\right) \leq 0.909$, and found the ferromagnetic-non-magnetic transition only for the case of 8 electrons on a $6 \times 2$ lattice, around $t_{\perp} /\left(t_{\perp}+t\right) \sim 0.45$. This is consistent with Kohno's the result 12 .

All these results indicate that the phase boundary between the ferromagnetic and the antiferromagnetic states is reasonably close to the thermodynamic limit for small systems when a care is taken (i.e., letting fully-polarized electrons take closed shell configurations).

\section{SUMMARY}

To summarize, we have studied the relation between the fully-polarized ferromagnetic state and the spiral spins state, a spin-singlet spin state that has a spin correlation length as large as the system size, which accompanies the fully-polarized ferromagnetic state in a number of electron correlation models. As a typical example, we calculate the energy of the SP state and the F state for one-dimensional $U=\infty t$ - $t^{\prime}$ Hubbard model, It suggests that the SP state is also the ground state in the thermodynamic limit when the F state is the ground state. Following the argument by Koma and Tasaki22, we have also indicated how a symmetry becomes broken in the SP state in the thermodynamic limit, where some 'low-lying states' become hybridized.

We have then characterized the SP state, an itinerant magnetic state, by calculating the one-particle spectral function. The result is interpreted in a picture in which holes move almost freely in a twisted spin configuration. We have also calculated the dynamical spin and charge correlation functions to find that their behaviors in the SP state are similar to those of the F state.

From these we have conjectured that we should regard the SP state and the F state are equivalent in the thermodynamic limit, even though the SP state (spin-singlet) and the F state (fully-polarized) are opposite extremes in terms of $\mathbf{S}_{\text {tot }}$. We have then shown that the magnetic phase diagram can be determined accurately from finite-size studies by taking appropriate boundary condition (that depends on the number of electrons which are accommodated in the shells in the non-interacting case) that pushes up the energy of the SP state. This enables us to concentrate on the F states, i.e., to simply look at the change in $S_{\text {tot }}$. This is permissible since the SP and F states are regarded to be equivalent, so that we can concentrate on either of them. We have obtained the phase diagram in this way for 1D $t-t^{\prime}$ Hubbard model, Tasaki's model and 2-leg Hubbard ladder. We have found that the phase boundary between the ferromagnetic state and the non-magnetic state determined in such a way accurately coincides with those obtained by the DMRG calculation for much larger systems. Since the method does not de- 
pend on the dimensionality, we may expect that it should be applicable to the determination of the phase diagram in two or higher dimensional systems.

\section{ACKNOWLEDGMENTS}

We would like to thank Kazuhiko Kuroki, Koichi Kusakabe, Tohru Koma for illuminating discussions. R.A. is supported by Japan Society for Promotion of Science. Numerical calculation were done on FACOM VPP $500 / 40$ at the Supercomputer center, Institute for Solid State Physics, University of Tokyo.

${ }^{1}$ M. C. Gutzwiller, Phys. Rev. Lett. 10,159 (1963).

${ }^{2}$ J. Hubbard, Proc. R. Soc. London, Ser. A 276, 238 (1963).

${ }^{3}$ J. Kanamori, Prog. Theor. Phys. 30, 275 (1963).

${ }^{4}$ Y. Nagaoka, Phys. Rev. 147, 392 (1966).

${ }^{5}$ A. Mielke, J. Phys. A24, L73 (1991).

${ }^{6}$ A. Mielke, J. Phys. A24, 3311 (1991).

${ }^{7}$ A. Mielke, J. Phys. A25, 4335 (1992).

${ }^{8}$ A. Mielke, Phys. Rev. Lett. 82, 4212 (1999).

${ }^{9}$ H. Tasaki, Phys. Rev. Lett. 69, 1608 (1992).

${ }^{10}$ A. Mielke and H. Tasaki, Commun. Math. Phys. 158, 31 (1993).

${ }^{11}$ H. Tasaki, Phys. Rev. Lett. 75, 4678 (1995).

12 M. Kohno, Phys. Rev. B 56, 15015 (1997).

${ }^{13}$ S. Daul and R. Noack, Z. Phys. B 103, 293 (1997).

${ }^{14}$ S. Daul and R. Noack, Phys. Rev. B 58, 2635 (1997).

${ }^{15}$ S. Liang and H. Pang, Europhys. Lett. 32, 173 (1995).

${ }^{16}$ H. Sakamoto and K. Kubo, J. Phys. Soc. Jpn. 65, 3732 (1996).

${ }^{17}$ K. Kusakabe and H. Aoki, Physica B 194-196, 217 (1994).

${ }^{18}$ K. Kusakabe and H. Aoki, Phys. Rev. B 52, R8684 (1995).

${ }^{19}$ Y. Watanabe and S. Miyashita, J. Phys. Soc. Jpn. 66, 3981 (1997).

${ }^{20}$ K. Kubo, J. Phys. Soc. Jpn. 51, 782 (1982).

${ }^{21}$ M. Sigrist, K. Ueda and H. Tsunetsugu, Phys. Rev. Lett 67, 2211 (1991).

${ }^{22}$ T. Koma and H. Tasaki, J. Stat. Phys. 76, 745 (1994).

${ }^{23}$ E. Müller-Hartmann, J. Low. Temp. Phys. 99, 349 (1995).

${ }^{24}$ R. Eder and Y. Ohta, Phys. Rev. B 56, R14247 (1997).

${ }^{25}$ R. Arita, K. Kusakabe, K. Kuroki and H. Aoki, Phys. Rev. B 58 R11833 (1998).

${ }^{26}$ B. Doucot and X.G. Wen, Phys. Rev. B 40, 2719 (1989).

FIG. 1. The DMRG result for the spin-spin correlation function for 24 electrons on a 32 -site $t$ - $t^{\prime}$ model with OBC.
FIG. 2. The DMRG result for the energy difference between the SP state and the ferromagnetic state, $\left.\Delta E \equiv E_{\mathrm{SP}}-E_{\mathrm{F}}\right)$, in $1 \mathrm{D} t-t^{\prime}$ Hubbard model as a function of inverse system size $1 / L$ for the density of electrons $n=0.5$ with the exchange interaction $J=0$ (solid lines), $J=0.2$ (dashed lines), or $J=-0.2$ (dotted lines).

FIG. 3. The energy difference between the SP state and the ferromagnetic state, $\Delta E \equiv E_{\mathrm{SP}}-E_{\mathrm{F}}$ ), as a function of inverse system size $1 / L$ for the density of electrons $n=0.5$ with the ordinary OBC (dashed line) or the boundary condition that omits the hopping at the both ends of the system (solid line).

FIG. 4. The spin-spin correlation function for 10 electrons on a 12 -site $t-t^{\prime}$ Hubbard model with $U=40$ and the periodic boundary condition.

FIG. 5. Single particle spectral function for 10 electrons on a 12 -site $t$ - $t^{\prime}$ Hubbard model with $U=40$. Solid lines denote the electron-removal spectrum, while dotted lines the electron-addition spectrum. Dashed curves indicate the energy dispersion defined by eq. (10).

FIG. 6. A plot similar to Fig. A for 6 electrons on a 12-site $t-t^{\prime}$ model with $U=4$ (solid line) and 6 (dashed line).

FIG. 7. A similar plot to Fig. Af for 6 electrons on a 12-site $t-t^{\prime}$ model with $U=6$.

FIG. 8. The ground state energy as a function of $1 / U$ for 6 electrons on a 12 -site $t$ - $t^{\prime}$ model.

FIG. 9. The dynamical spin(a) and charge(b) correlation functions for the SP state (dashed line) and the F state (solid line) for 10 electrons on a 12-site 1D $t-t^{\prime}$ Hubbard model with $U=40$.

FIG. 10. The spin-spin correlation function for 10 electrons on a 12-site 1D Tasaki's model for $U=4,6$.

FIG. 11. A plot similar to Fig. 5 for 6 electrons on a 12 -site 1D Tasaki's model for $U=12$. We have superposed the results when we take $\gamma_{i, \sigma}^{+}=c_{2 i, \sigma}^{\dagger}$ and $\gamma_{i, \sigma}^{+}=c_{2 i-1, \sigma}^{\dagger}$. Dashed curves indicate the dispersion defined by eq. (12).

FIG. 12. The spin-spin correlation function for 10 electrons on a $8 \times 2 U=\infty$ Hubbard ladder. 
FIG. 13. A similar plot to Fig. 5 for 14 electrons on a $8 \times 2$ ladder. Dashed curves indicate the dispersion defined by eq. (11).

FIG. 14. Phase diagram of 1D $t-t^{\prime}$ Hubbard model for $t^{\prime}=0.2$ obtained by exact diagonalization for a 12 -site system(diamonds) 10-site system(circles). A DMRG result of Daul and Noack 13,14 is superposed (a full line).
FIG. 15. A plot similar to Fig. 14, for the $t-t^{\prime}$ Hubbard model with $t^{\prime}=0.8$.

FIG. 16. Phase diagram against $U$ and $\beta$ for 1D Tasaki's model with $n=1 / 2$ for a 12 -site system(diamonds) or 8 -site syste (circles) with PBC. A DMRG result by Sakamoto and Kubd 16 are superposed (a solid line). 
This figure "fig1.gif" is available in "gif" format from: http://arxiv.org/ps/cond-mat/0002442v1 
This figure "fig2.gif" is available in "gif" format from: http://arxiv.org/ps/cond-mat/0002442v1 
This figure "fig3.gif" is available in "gif" format from: http://arxiv.org/ps/cond-mat/0002442v1 
This figure "fig4.gif" is available in "gif" format from: http://arxiv.org/ps/cond-mat/0002442v1 
This figure "fig5.gif" is available in "gif" format from: http://arxiv.org/ps/cond-mat/0002442v1 
This figure "fig6.gif" is available in "gif" format from: http://arxiv.org/ps/cond-mat/0002442v1 
This figure "fig7.gif" is available in "gif" format from: http://arxiv.org/ps/cond-mat/0002442v1 
This figure "fig8.gif" is available in "gif" format from: http://arxiv.org/ps/cond-mat/0002442v1 
This figure "fig9a.gif" is available in "gif" format from: http://arxiv.org/ps/cond-mat/0002442v1 
This figure "fig9b.gif" is available in "gif" format from: http://arxiv.org/ps/cond-mat/0002442v1 
This figure "fig10.gif" is available in "gif" format from: http://arxiv.org/ps/cond-mat/0002442v1 
This figure "fig11.gif" is available in "gif" format from: http://arxiv.org/ps/cond-mat/0002442v1 
This figure "fig12.gif" is available in "gif" format from: http://arxiv.org/ps/cond-mat/0002442v1 
This figure "fig13.gif" is available in "gif" format from: http://arxiv.org/ps/cond-mat/0002442v1 
This figure "fig14.gif" is available in "gif" format from: http://arxiv.org/ps/cond-mat/0002442v1 
This figure "fig15.gif" is available in "gif" format from: http://arxiv.org/ps/cond-mat/0002442v1 
This figure "fig16.gif" is available in "gif" format from: http://arxiv.org/ps/cond-mat/0002442v1 